\title{
Does inflammation help during COVID-19?
}

\author{
To the Editor:
}

Coronavirus disease 2019 (COVID-19) has emerged as the most devastating pandemic in the last 100 years and has already killed almost 1 million people [1] despite the most aggressive efforts to contain the infection in recorded history. Currently, it remains unclear what drives disease severity in COVID-19, limiting therapeutic options. Various immune modulators such as glucocorticoids and anti-inflammatory therapies are being used to treat COVID-19 without clear evidence of inflammation playing a detrimental role. Here we review evidence to suggest that the host's ability to clear the viral infection is the major determinant in the host survival where a robust early inflammatory response in the lung provides a survival advantage to the host by aiding effective viral clearance. However, failure to clear the viral infection leads to an exacerbated inflammatory response at a later time point, which becomes detrimental to the host's survival. This concept can explain the high susceptibility associated with advanced age or male sex and at the same time explains why children are largely spared of this lethal disease but have inflammatory manifestations such as multi-system inflammatory syndrome in children (MIS-C) or Kawasaki-like disease.

The most susceptible population to COVID-19 remains the aged population which is known to mount a poor inflammatory response against a wide range of respiratory pathogens [2]. Moreover, this population tends to have poor vaccine responses and require additional adjuvants to induce a sufficient immune response [3]. The lack of targeted inflammatory response in lung to the viral infection in the aged population should not be confused with overall elevated levels of inflammatory cytokines at the baseline in older subjects, a phenomenon often referred to as "inflammaging" [4].

In contrast to the elderly, children seem to be protected from the disease. This differs from influenza, which effectively infects children and causes significant morbidity and mortality, especially in low-income countries. The precise reasons for this protection remain unknown but may be multifactorial. The lack of comorbidities or the presence of a robust innate inflammatory response has been speculated. Surprisingly, an interesting phenomenon was described in some children who successfully recovered from COVID-19 but then presented with MIS-C. The level of the inflammatory cytokine interleukin- 6 was elevated in a significant percentage of these children [5]. Interestingly, this inflammatory disease is not associated with the early disease severity in these children, and many of them were only found to be infected with severe acute respiratory syndrome coronavirus 2 (SARS-CoV-2) by antibody testing [5]. These cases hint that children may have a robust early inflammatory response that allows them to effectively clear the invading virus to limit disease manifestation, but this potentially makes them more prone to inflammatory sequelae such as MIS-C.

The female sex is protected against SARS-CoV-2 compared to the male counterpart in terms of both susceptibility and severity of the disease with an unknown biological basis. It is well known that females have a high inflammatory response and increased vaccine response that often leads to an excessive site of injection reaction among other side effects [6]. Here, it is again possible that a robust early inflammatory response in females is contributing to the limited disease severity.

The major question that remains unclear is what exactly drives the disease severity and lethality in the vulnerable population in COVID-19. A diverse set of comorbidities have been identified that render the population more susceptible to infections. It is not known whether these comorbidities converge at a single biological event that renders patients vulnerable. Our recent study shows that patients with

@ERSpublications

Persistent viral presence, rather than the early exaggerated inflammation, may be a key determinant of disease severity in \#COVID19. If so, the focus of new therapies should be to promote viral clearance to decrease disease severity and mortality. https://bit.ly/3ahgzkP

Cite this article as: Sharma L, Chang D, Dela Cruz CS. Does inflammation help during COVID-19?. ERJ Open Res 2020; 6: 00557-2020 [https://doi.org/10.1183/23120541.00557-2020]. 
persistent viral presence have a significantly worse disease and disease-associated outcomes, suggesting impaired viral clearance as a key underlying factor that determines disease severity [7]. Similarly, others have found that severe cases have much higher viral load and prolonged viral persistence compared to mild infections [8]. The strongest evidence to support the role of persistent viral infections in mediating the lethality in COVID-19 comes from the deceased patients who maintain their viral positivity until their death [9].

Further strong evidence suggesting the role of viral infection in mediating the disease is the depletion of antiviral immune cells. The depletion is specific to the T-cells, which include both helper CD4 ${ }^{+}$cells and cytotoxic $\mathrm{CD}^{+} \mathrm{T}$-cells. The depletion of $\mathrm{T}$-cells has been reported by almost all studies comparing the disease severity in patients, including ours $[7,9]$. Of significance, the depletion in the T-cell population is observed long before other markers of disease severity are observed, suggesting T-cell depletion directly contributes to the impaired antiviral immune response. We believe that impaired antiviral immunity leads to persistent viral presence and associated disease severity.

Some researchers have been suggesting the role of inflammatory cytokines in mediating the disease severity, a term often referred to as "cytokine storm". We believe that elevated inflammation is more likely a consequence of the uncontrolled viral infection. Most of the data suggest that an increase in inflammation occurs at later stages of the disease, which may be mediated by damage that is already caused by persistent viral replication in the lung. Inhibiting inflammation at an early time point may be detrimental to the patient's ability to effectively clear the infection and can be counterproductive in limiting the disease severity. A recent large-scale study using dexamethasone supports this notion where dexamethasone improved disease outcome in patients with mechanical ventilation but impaired the host recovery in patients who were relatively healthy and were not on oxygen therapy [10]. The effectiveness of anti-inflammatory therapies in later stages of the disease may also be of limited value as active viral replication continues in patients for prolonged periods and until death in those who succumb to the infection. Inhibition of inflammation may be more suitable for patient populations who effectively clear the viral infection or could be used alongside effective antiviral agents. Inhibition of inflammation could also be beneficial in those who have chronic consequences of exaggerated inflammatory responses such as children, which may help avoid inflammatory sequelae such as MIS-C.

Here we suggest that persistent viral presence rather than early exaggerated inflammation is a key determinant of the disease severity in COVID-19 (figure 1). The primary focus of new therapies should be to promote viral clearance to decrease disease severity and subsequent mortality. The use of anti-inflammatory therapies should be reserved only for those who can clear the virus infection, in

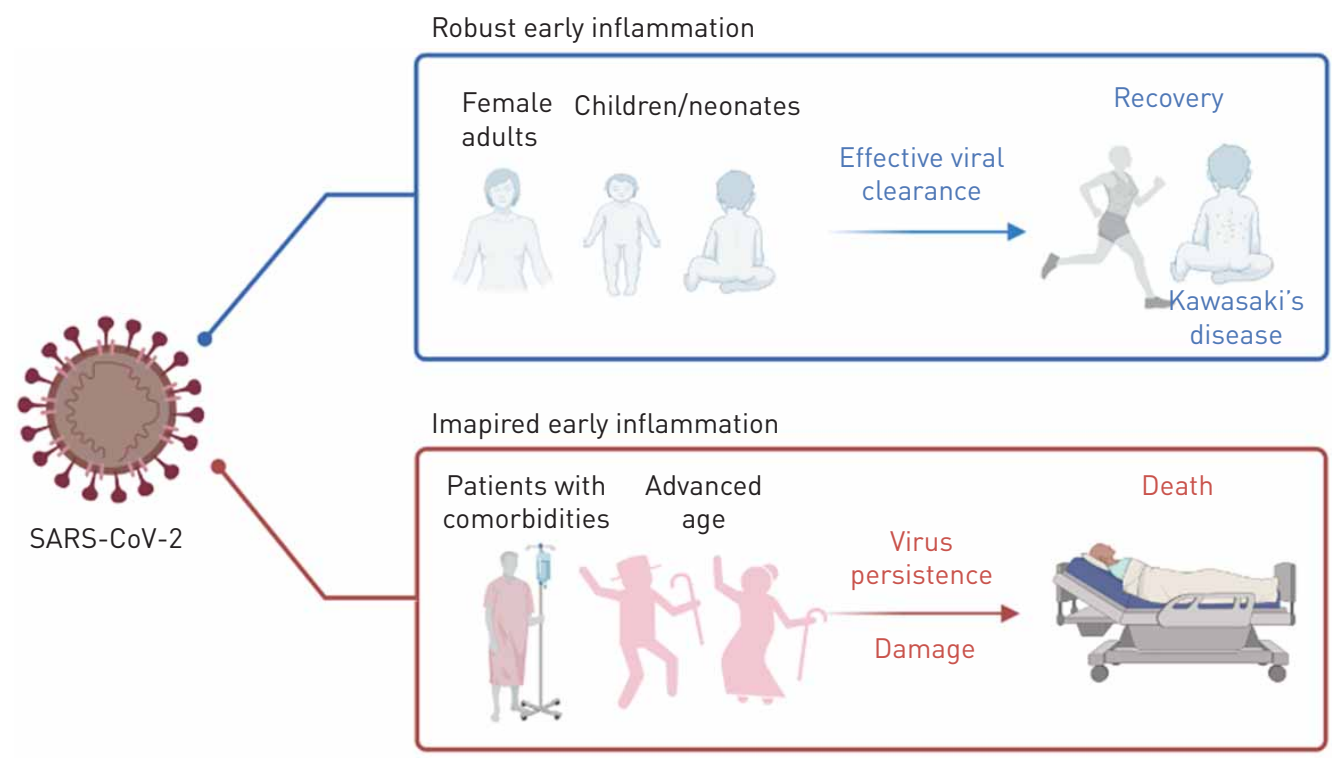

FIGURE 1 Patient outcomes of severe acute respiratory syndrome coronavirus 2 (SARS-CoV-2) infection depends on the early inflammatory response. Children and females are more protected due to their ability to mount an early robust inflammatory response, but this makes children susceptible to inflammatory sequelae. The impaired early inflammatory response in older adults or patients with comorbidities leads to viral persistence resulting in severe disease and possibly death. 
conjunction with direct antiviral agents, or where inhibiting the inflammatory response outweighs the risk of impairing viral clearance.

Lokesh Sharma $^{1,3}$, De Chang ${ }^{2,3}$ and Charles S. Dela Cruz ${ }^{1,3}$

${ }^{1}$ Section of Pulmonary and Critical Care and Sleep Medicine, Dept of Medicine, Yale University School of Medicine, New Haven, USA. ${ }^{2}$ Third Medical Center of Chinese PLA General Hospital, Beijing, China. ${ }^{3}$ All authors contributed equally.

Correspondence: Lokesh Sharma, Charles S Dela Cruz, Section of Pulmonary and Critical Care and Sleep Medicine, TAC S-440, 300 Cedar Street, New Haven, CT, USA, E-mail: lokeshkumar.sharma@yale.edu.

Received: 6 Aug 2020 | Accepted: 6 Aug 2020

Conflict of interest: None declared.

\section{Reference}

1 World Health Organization. Coronavirus Disease (COVID-19): Weekly Epidemiological Update. https://www.who. int/docs/default-source/coronaviruse/situation-reports/20200914-weekly-epi-update-5.pdf?sfvrsn=cf929d04_2 Date last accessed: 17 September 2020.

2 Metcalf TU, Cubas RA, Ghneim K, et al. Global analyses revealed age-related alterations in innate immune responses after stimulation of pathogen recognition receptors. Aging Cell 2015; 14: 421-432.

3 Crooke SN, Ovsyannikova IG, Poland GA, et al. Immunosenescence and human vaccine immune responses. Immun Ageing 2019; 16: 25.

4 Franceschi C, Capri M, Monti D, et al. Inflammaging and anti-inflammaging: a systemic perspective on aging and longevity emerged from studies in humans. Mech Ageing Dev 2007; 128: 92-105.

5 Verdoni L, Mazza A, Gervasoni A, et al. An outbreak of severe Kawasaki-like disease at the Italian epicentre of the SARS-CoV-2 epidemic: an observational cohort study. Lancet 2020; 395: 1771-1778.

6 Furman D, Hejblum BP, Simon N, et al. Systems analysis of sex differences reveals an immunosuppressive role for testosterone in the response to influenza vaccination. Proc Natl Acad Sci 2014; 111: 869-874.

7 Chang D, Zhao P, Zhang D-W, et al. Persistent viral presence determines the clinical course of the disease in COVID-19. J Allergy Clin Immunol Practice 2020; 8: 2585-2591.

8 Liu Y, Yan L-M, Wan L, et al. Viral dynamics in mild and severe cases of COVID-19. Lancet Infect Dis 2020; 20 656-657.

9 Zhou F, Yu T, Du R, et al. Clinical course and risk factors for mortality of adult inpatients with COVID-19 in Wuhan, China: a retrospective cohort study. Lancet 2020; 395: 1054-1062.

10 Horby P, Lim WS, Emberson J, et al. Effect of dexamethasone in hospitalized patients with COVID-19: preliminary report. medRxiv 2020; preprint [https://doi.org/10.1101/2020.06.22.20137273]. 